\title{
NUMERICAL ANALYSIS OF A FRICTIONLESS VISCOELASTIC PIEZOELECTRIC CONTACT PROBLEM*
}

\author{
Mikael Barboted ${ }^{1}$, Jose Ramon Fernández ${ }^{2}$ and Youssef OuafiK ${ }^{1}$
}

\begin{abstract}
In this work, we consider the quasistatic frictionless contact problem between a viscoelastic piezoelectric body and a deformable obstacle. The linear electro-viscoelastic constitutive law is employed to model the piezoelectric material and the normal compliance condition is used to model the contact. The variational formulation is derived in a form of a coupled system for the displacement and electric potential fields. An existence and uniqueness result is recalled. Then, a fully discrete scheme is introduced based on the finite element method to approximate the spatial variable and an Euler scheme to discretize the time derivatives. Error estimates are derived on the approximative solutions and, as a consequence, the linear convergence of the algorithm is deduced under suitable regularity conditions. Finally, some two-dimensional examples are presented to demonstrate the performance of the algorithm.
\end{abstract}

Mathematics Subject Classification. 65N15, 65N30, 74D10, 74M15, 74S05, 74S20.

Received July 23, 2007. Revised January 18, 2008.

Published online June 5, 2008.

\section{INTRODUCTION}

In this work, we study, from the numerical point of view, a frictionless contact problem between a viscoelastic piezoelectric body and a deformable obstacle.

Piezoelectricity is the ability of certain crystals, like the quartz (also ceramics $\left(\mathrm{BaTiO}_{3}, \mathrm{KNbO}_{3}, \mathrm{LiNbO}_{3}\right.$, etc.) and even the human mandible or the human bone), to produce a voltage when they are subjected to mechanical stress. On a nanoscopic scale, the piezoelectric phenomenon arises from a nonuniform charge distribution within a crystal unit cells. When such a crystal is mechanically deformed, the positive and negative charge centers displace by differing amounts. Thus, while the overall crystal remains electrically neutral, the difference in charge center displacement results in an electric polarization within the crystal. Electric polarization due to mechanical input is perceived as piezoelectricity.

The piezoelectric effect is characterized by the coupling between the mechanical and the electrical properties of the material: it was observed that the appearance of electric charges on some crystals was due to the action

\footnotetext{
Keywords and phrases. Piezoelectricity, viscoelasticity, normal compliance, error estimates, numerical simulations.

* The work of the second author was partially supported by the Ministerio de Educación y Ciencia (Project MTM2006-13981).

${ }^{1}$ Laboratoire de Mathématiques et Physique pour les Systèmes (MEPS), Bâtiment B3, case courrier 12, 52 Avenue Paul Alduy, 66860 Perpignan, France. barboteu@univ-perp.fr; youssef.ouafik@univ-perp.fr

2 Departamento de Matemática Aplicada, Facultade de Matemáticas, Campus Sur s/n, Universidade de Santiago de Compostela, 15782 Santiago de Compostela, Spain. jramon@usc.es
} 


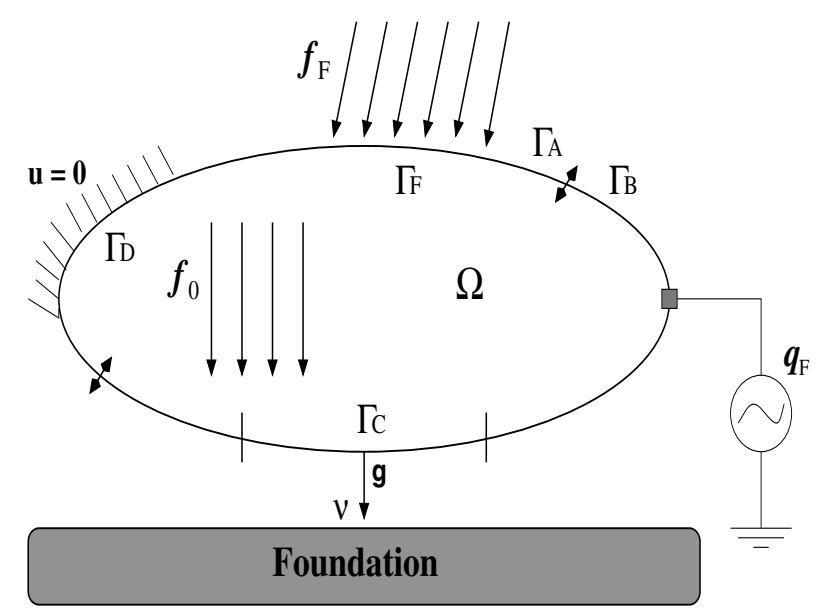

FIgURE 1. A viscoelastic piezoelectric body in contact with a foundation.

of body forces and surface fractions and, conversely, the action of the electric field generated strain or stress in the body. This kind of materials appears usually in the industry as switches in radiotronics, electroacoustics or measuring equipments.

Different models have been developed early to describe the interaction between the electric and mechanical fields (see, e.g., $[2,4,13,17-20,25-28]$ and the references therein). Recently, contact problems involving elasticpiezoelectric materials $[3,5,12,15,21,23,24]$ or viscoelastic piezoelectric materials [22] have been studied.

In this paper, we consider a viscoelastic piezoelectric body which may become in contact with a deformable obstacle, the so-called foundation. The contact is assumed frictionless and a normal compliance condition is employed to model it (see [14,16]). This paper continues [22], providing the numerical analysis of the variational problem and some numerical results which exhibit its behaviour, and extends the results of [3,12] to the case of viscoelastic materials.

The rest of the paper is structured as follows. In Section 2 we present the mechanical model, provide its variational formulation and state an existence and uniqueness result, Theorem 2.1. Then, a fully discrete scheme is introduced in Section 3 based on the finite element method to approximate the spatial variable and an Euler scheme to discretize the time derivatives. A main error estimates result is proved, Theorem 3.3, from which the linear convergence of the algorithm is deduced under suitable regularity conditions (see Cor. 3.4). Finally, some numerical examples are presented in Section 4 in order to show the performance of the method.

\section{MechanicAl PROBlem AND its VARIATIONAL FORMUlation}

Denote by $\mathbb{S}^{d}$ the space of second order symmetric tensors on $\mathbb{R}^{d}$ and by "." and $\|\cdot\|$ the inner product and the Euclidean norms on $\mathbb{R}^{d}$ and $\mathbb{S}^{d}$.

Let $\Omega \subset \mathbb{R}^{d}, d=1,2,3$, denote a domain occupied by a viscoelastic piezoelectric body with a smooth boundary $\Gamma=\partial \Omega$. We denote by $\boldsymbol{\nu}$ the unit outer normal vector to $\Gamma$ and we assume that this boundary is decomposed into three measurable parts $\Gamma_{D}, \Gamma_{F}, \Gamma_{C}$, on one hand, and on two measurable parts $\Gamma_{A}$ and $\Gamma_{B}$, on the other hand, such that meas $\left(\Gamma_{D}\right)>0$, meas $\left(\Gamma_{A}\right)>0$, and $\Gamma_{C} \subseteq \Gamma_{B}$. Finally, let $[0, T], T>0$, be the time interval of interest (see Fig. 1).

Let $\boldsymbol{x} \in \Omega$ and $t \in[0, T]$ be the spatial and time variables, respectively, and, in order to simplify the writing, we do not indicate the dependence of the functions on $\boldsymbol{x}$ and $t$. Moreover, a dot above a variable represents the derivative with respect to the time variable. 
Let us denote by $\boldsymbol{u}$ the displacement field, $\boldsymbol{\sigma}$ the stress tensor, $\boldsymbol{\varepsilon}(\boldsymbol{u})=\left(\varepsilon_{i j}(\boldsymbol{u})\right)_{i, j=1}^{d}$ the linearized strain tensor given by

and $\varphi$ the electric potential.

$$
\varepsilon_{i j}(\boldsymbol{u})=\frac{1}{2}\left(\frac{\partial u_{i}}{\partial x_{j}}+\frac{\partial u_{j}}{\partial x_{i}}\right)
$$

The body is assumed viscoelastic piezoelectric and satisfying the following constitutive law (see $[7,22])$,

$$
\boldsymbol{\sigma}=\mathcal{A} \varepsilon(\dot{\boldsymbol{u}})+\mathcal{B} \varepsilon(\boldsymbol{u})-\mathcal{E}^{*} \mathbf{E}(\varphi)
$$

where $\mathcal{A}$ and $\mathcal{B}$ are the fourth-order viscosity and elastic tensors, respectively, $\mathbf{E}(\varphi)=\left(E_{i}(\varphi)\right)_{i=1}^{d}$ represents the electric field defined by

$$
E_{i}(\varphi)=-\frac{\partial \varphi}{\partial x_{i}}, \quad i=1, \ldots, d
$$

and $\mathcal{E}^{*}=\left(e_{i j k}^{*}\right)_{i, j, k=1}^{d}$ denotes the transpose of the third-order piezoelectric tensor $\mathcal{E}=\left(e_{i j k}\right)_{i, j, k=1}^{d}$. We recall that

$$
e_{i j k}^{*}=e_{k i j}, \quad \text { for all } i, j, k=1, \ldots, d .
$$

Following [4] the following constitutive law is satisfied for the electric potential,

$$
\mathbf{D}=\mathcal{E} \varepsilon(\boldsymbol{u})+\beta \mathbf{E}(\varphi)
$$

where $\mathbf{D}$ is the electric displacement field and $\beta$ is the electric permittivity tensor.

Since the process is assumed quasistatic, the inertia effects are negligible and therefore,

$$
\begin{aligned}
& \operatorname{Div} \boldsymbol{\sigma}+\boldsymbol{f}_{0}=\mathbf{0} \quad \text { in } \quad \Omega \times(0, T), \\
& \operatorname{div} \mathbf{D}=q_{0} \quad \text { in } \quad \Omega \times(0, T),
\end{aligned}
$$

where $\boldsymbol{f}_{0}$ is the density of the body forces acting in $\Omega$ and $q_{0}$ is the volume density of free electric charges. Moreover, Div and div represent the divergence operators for tensor and vector functions, respectively.

We turn now to describe the boundary conditions.

On the boundary part $\Gamma_{D}$ we assume that the body is clamped and thus the displacement field neglects there, that is $\boldsymbol{u}=\mathbf{0}$ on $\Gamma_{D} \times(0, T)$. Moreover, we assume that a density of traction forces, denoted by $\boldsymbol{f}_{F}$, acts on the boundary part $\Gamma_{F}$, i.e.,

$$
\boldsymbol{\sigma} \boldsymbol{\nu}=\boldsymbol{f}_{F} \quad \text { on } \quad \Gamma_{F} \times(0, T) .
$$

On the part $\Gamma_{C}$ the body can become in contact with a deformable insulator obstacle, the so-called foundation. According to [14] the following normal compliance contact condition is employed,

$$
-\sigma_{\nu}=p\left(u_{\nu}-g\right) \text { on } \Gamma_{C} \times(0, T),
$$

where $\sigma_{\nu}=\boldsymbol{\sigma} \boldsymbol{\nu} \cdot \boldsymbol{\nu}$ is the normal stress, $u_{\nu}=\boldsymbol{u} \cdot \boldsymbol{\nu}$ denotes the normal displacement, $g$ represents the gap between the body and the obstacle measured along the normal direction $\nu$ and $p$ is a given function whose properties will be described below. Finally, we assume that the contact is frictionless and therefore, $\boldsymbol{\sigma}_{\tau}=\boldsymbol{\sigma} \boldsymbol{\nu}-\sigma_{\nu} \boldsymbol{\nu}=\mathbf{0}$.

Let $\Omega$ be subject to a prescribed electric potential $\varphi_{A}$ on $\Gamma_{A}$ and to a density of surface electric charges $q_{F}$ on $\Gamma_{B}$, that is,

$$
\begin{aligned}
& \varphi=\varphi_{A} \quad \text { on } \quad \Gamma_{A} \times(0, T), \\
& \mathbf{D} \cdot \boldsymbol{\nu}=q_{F} \quad \text { on } \quad \Gamma_{B} \times(0, T) .
\end{aligned}
$$

We assume that $q_{F}=0$ on $\Gamma_{C}$, that is, the foundation is supposed to be insulator. We note that it is straightforward to extend the results presented below to more general situations by decomposing $\Gamma$ in a different way.

The mechanical problem of the quasistatic contact of a viscoelastic piezoelectric body with a deformable obstacle is then written as follows. 
Problem P. Find a displacement field $\boldsymbol{u}: \Omega \times(0, T) \rightarrow \mathbb{R}^{d}$, a stress field $\boldsymbol{\sigma}: \Omega \times(0, T) \rightarrow \mathbb{S}^{d}$, an electric potential field $\varphi: \Omega \times(0, T) \rightarrow \mathbb{R}$ and an electric displacement field $\mathbf{D}: \Omega \times(0, T) \rightarrow \mathbb{R}^{d}$ such that,

$$
\begin{aligned}
& \boldsymbol{\sigma}=\mathcal{A} \varepsilon(\dot{\boldsymbol{u}})+\mathcal{B} \varepsilon(\boldsymbol{u})-\mathcal{E}^{*} \mathbf{E}(\varphi) \quad \text { in } \quad \Omega \times(0, T), \\
& \mathbf{D}=\mathcal{E} \varepsilon(\boldsymbol{u})+\beta \mathbf{E}(\varphi) \quad \text { in } \quad \Omega \times(0, T), \\
& \operatorname{Div} \boldsymbol{\sigma}+\boldsymbol{f}_{0}=\mathbf{0} \quad \text { in } \quad \Omega \times(0, T), \\
& \operatorname{div} \mathbf{D}=q_{0} \quad \text { in } \quad \Omega \times(0, T), \\
& \boldsymbol{u}=\mathbf{0} \quad \text { on } \Gamma_{D} \times(0, T), \\
& \boldsymbol{\sigma} \boldsymbol{\nu}=\boldsymbol{f}_{F} \quad \text { on } \quad \Gamma_{F} \times(0, T), \\
& \boldsymbol{\sigma}_{\tau}=\mathbf{0}, \quad-\sigma_{\nu}=p\left(u_{\nu}-g\right) \quad \text { on } \quad \Gamma_{C} \times(0, T), \\
& \varphi=\varphi_{A} \quad \text { on } \quad \Gamma_{A} \times(0, T), \\
& \mathbf{D} \cdot \boldsymbol{\nu}=q_{F} \quad \text { on } \quad \Gamma_{B} \times(0, T), \\
& \boldsymbol{u}(0)=\boldsymbol{u}_{0} \quad \text { in } \quad \Omega \text {. }
\end{aligned}
$$

Here, $\boldsymbol{u}_{0}$ represents an initial condition for the displacement field.

In order to obtain the variational formulation of Problem $\mathrm{P}$, let us introduce the variational spaces $V, Q$ and $W$, and the convex set $W_{A}$ as follows,

$$
\begin{aligned}
& V=\left\{\boldsymbol{v} \in\left[H^{1}(\Omega)\right]^{d} ; \boldsymbol{v}=\mathbf{0} \quad \text { on } \quad \Gamma_{D}\right\}, \\
& Q=\left\{\boldsymbol{\tau}=\left(\tau_{i j}\right)_{i, j=1}^{d} \in\left[L^{2}(\Omega)\right]^{d \times d} ; \tau_{i j}=\tau_{j i}, i, j=1, \ldots, d\right\}, \\
& W=\left\{\psi \in H^{1}(\Omega) ; \psi=0 \quad \text { on } \quad \Gamma_{A}\right\}, \\
& W_{A}=\left\{\psi \in H^{1}(\Omega) ; \psi=\varphi_{A} \quad \text { on } \quad \Gamma_{A}\right\}
\end{aligned}
$$

and denote by $H=\left[L^{2}(\Omega)\right]^{d}$.

The viscosity tensor $\mathcal{A}(\boldsymbol{x})=\left(a_{i j k l}(\boldsymbol{x})\right)_{i, j, k, l=1}^{d}: \boldsymbol{\tau} \in \mathbb{S}^{d} \rightarrow \mathcal{A}(\boldsymbol{x})(\boldsymbol{\tau}) \in \mathbb{S}^{d}$ satisfies:

(a) $a_{i j k l}=a_{k l i j}=a_{j i k l}$ for $i, j, k, l=1, \ldots, d$.

(b) $a_{i j k l} \in L^{\infty}(\Omega)$ for $i, j, k, l=1, \ldots, d$.

(c) There exists $m_{\mathcal{A}}>0$ such that $\mathcal{A}(\boldsymbol{x}) \boldsymbol{\tau} \cdot \boldsymbol{\tau} \geq m_{\mathcal{A}}\|\boldsymbol{\tau}\|^{2}$

$$
\forall \boldsymbol{\tau} \in \mathbb{S}^{d} \text {, a.e. } \boldsymbol{x} \in \Omega \text {. }
$$


The elastic tensor $\mathcal{B}(\boldsymbol{x})=\left(b_{i j k l}(\boldsymbol{x})\right)_{i, j, k, l=1}^{d}: \boldsymbol{\tau} \in \mathbb{S}^{d} \rightarrow \mathcal{B}(\boldsymbol{x})(\boldsymbol{\tau}) \in \mathbb{S}^{d}$ verifies:

$$
\begin{aligned}
& \text { (a) } b_{i j k l}=b_{k l i j}=b_{j i k l} \text { for } i, j, k, l=1, \ldots, d \text {. } \\
& \text { (b) } b_{i j k l} \in L^{\infty}(\Omega) \text { for } i, j, k, l=1, \ldots, d \text {. }
\end{aligned}
$$

The piezoelectric tensor $\mathcal{E}(\boldsymbol{x})=\left(e_{i j k}(\boldsymbol{x})\right)_{i, j, k=1}^{d}: \boldsymbol{\tau} \in \mathbb{S}^{d} \rightarrow \mathcal{E}(\boldsymbol{x})(\boldsymbol{\tau}) \in \mathbb{R}^{d}$ satisfies:

$$
\begin{aligned}
& \text { (a) } e_{i j k}=e_{i k j} \quad \text { for } \quad i, j, k=1, \ldots, d \text {. } \\
& \text { (b) } e_{i j k} \in L^{\infty}(\Omega) \text { for } i, j, k=1, \ldots, d \text {. }
\end{aligned}
$$

The permittivity tensor $\beta(\boldsymbol{x})=\left(\beta_{i j}(\boldsymbol{x})\right)_{i, j, k, l=1}^{d}: \boldsymbol{w} \in \mathbb{R}^{d} \rightarrow \beta(\boldsymbol{x})(\boldsymbol{w}) \in \mathbb{R}^{d}$ verifies:

$$
\begin{aligned}
& \text { (a) } \beta_{i j}=\beta_{j i} \text { for } i, j=1, \ldots, d \text {. } \\
& \text { (b) } \beta_{i j} \in L^{\infty}(\Omega) \text { for } i, j=1, \ldots, d \text {. } \\
& \text { (c) There exists } m_{\beta}>0 \text { such that } \beta(\boldsymbol{x}) \boldsymbol{w} \cdot \boldsymbol{w} \geq m_{\beta}\|\boldsymbol{w}\|^{2} \\
& \forall \boldsymbol{w} \in \mathbb{R}^{d} \text {, a.e. } \boldsymbol{x} \in \Omega \text {. }
\end{aligned}
$$

The normal compliance function $p(\boldsymbol{x}): r \in \mathbb{R} \rightarrow p(\boldsymbol{x}, r) \in[0, \infty)$ satisfies:

(a) There exists $m_{p}>0$ such that

$$
\begin{gathered}
\left|p\left(\boldsymbol{x}, r_{1}\right)-p\left(\boldsymbol{x}, r_{2}\right)\right| \leq m_{p}\left|r_{1}-r_{2}\right| \\
\forall r_{1}, r_{2} \in \mathbb{R}, \text { a.e. } \boldsymbol{x} \in \Gamma_{C} .
\end{gathered}
$$

(b) $\left(p\left(\boldsymbol{x}, r_{1}\right)-p\left(\boldsymbol{x}, r_{2}\right)\right)\left(r_{1}-r_{2}\right) \geq 0 \quad \forall r_{1}, r_{2} \in \mathbb{R}$, a.e. $\boldsymbol{x} \in \Gamma_{C}$.

(c) The mapping $\boldsymbol{x} \in \Gamma_{C} \mapsto p(\boldsymbol{x}, r)$ is measurable on $\Gamma_{C}$, for all $r \in \mathbb{R}$.

(d) $p(\boldsymbol{x}, r)=0$ for all $r \leq 0$.

The following regularity is assumed on the density of volume forces, tractions, volume electric charges and surface electric charges:

$$
\begin{aligned}
& \boldsymbol{f}_{0} \in C([0, T] ; H), \quad \boldsymbol{f}_{F} \in C\left([0, T] ;\left[L^{2}\left(\Gamma_{F}\right)\right]^{d}\right), \\
& q_{0} \in C\left([0, T] ; L^{2}(\Omega)\right), \quad q_{F} \in C\left([0, T] ; L^{2}\left(\Gamma_{B}\right)\right) .
\end{aligned}
$$

Finally, we assume that the gap function, the initial displacements and the boundary condition $\varphi_{A}$ satisfy

$$
g \in L^{2}\left(\Gamma_{C}\right), \quad g(\boldsymbol{x}) \geq 0 \quad \text { a.e. } \boldsymbol{x} \in \Gamma_{C}, \quad \boldsymbol{u}_{0} \in V, \quad \varphi_{A} \in C\left([0, T], C\left(\overline{\Gamma_{A}}\right)\right) .
$$

Using the Riesz' Theorem, we define the linear mappings $f:[0, T] \rightarrow V$ and $q:[0, T] \rightarrow W$ as follows,

$$
\begin{aligned}
& (\boldsymbol{f}(t), \boldsymbol{w})_{V}=\int_{\Omega} \boldsymbol{f}_{0}(t) \cdot \boldsymbol{w} \mathrm{d} \boldsymbol{x}+\int_{\Gamma_{F}} \boldsymbol{f}_{F}(t) \cdot \boldsymbol{w} \mathrm{d} \Gamma, \quad \forall \boldsymbol{w} \in V, \\
& (q(t), \psi)_{W}=\int_{\Omega} q_{0}(t) \psi \mathrm{d} \boldsymbol{x}+\int_{\Gamma_{B}} q_{F}(t) \psi \mathrm{d} \Gamma, \quad \forall \psi \in W .
\end{aligned}
$$

We notice that regularity assumptions (2.16) imply that $f \in C([0, T] ; V)$ and $q \in C([0, T] ; W)$.

Let us denote by $j: V \times V \rightarrow \mathbb{R}$ the normal compliance functional given by

$$
j(\boldsymbol{u}, \boldsymbol{v})=\int_{\Gamma_{C}} p\left(u_{\nu}-g\right) v_{\nu} \mathrm{d} \Gamma, \quad \forall \boldsymbol{u}, \boldsymbol{v} \in V,
$$

where, for all $\boldsymbol{v} \in V$, we let $v_{\nu}=\boldsymbol{v} \cdot \boldsymbol{\nu}$.

Plugging (2.1) into (2.3) and (2.2) into (2.4), keeping in mind that $\mathbf{E}(\varphi)=-\nabla \varphi$ and using the boundary conditions (2.5)-(2.9), applying a Green's formula we derive the following variational formulation of Problem P. 
Problem VP. Find a displacement field $\boldsymbol{u}:[0, T] \rightarrow V$ and an electric potential field $\varphi:[0, T] \rightarrow W_{A}$ such that $\boldsymbol{u}(0)=\boldsymbol{u}_{0}$ and for all $t \in[0, T]$,

$$
\begin{gathered}
(\mathcal{A} \varepsilon(\dot{\boldsymbol{u}}(t)), \boldsymbol{\varepsilon}(\boldsymbol{w}))_{Q}+(\mathcal{B} \boldsymbol{\varepsilon}(\boldsymbol{u}(t)), \boldsymbol{\varepsilon}(\boldsymbol{w}))_{Q}+\left(\mathcal{E}^{*} \nabla \varphi(t), \boldsymbol{\varepsilon}(\boldsymbol{w})\right)_{Q}+j(\boldsymbol{u}(t), \boldsymbol{w}) \\
=(\boldsymbol{f}(t), \boldsymbol{w})_{V}, \quad \forall \boldsymbol{w} \in V, \\
(\beta \nabla \varphi(t), \nabla \psi)_{H}-(\mathcal{E} \boldsymbol{\varepsilon}(\boldsymbol{u}(t)), \nabla \psi)_{H}=(q(t), \psi)_{W}, \quad \forall \psi \in W .
\end{gathered}
$$

Using analogous ideas to those employed in [21] for a normal compliance elastic problem or in [22] for the case of viscoelastic materials, we obtain the following theorem which states the existence of a unique weak solution to Problem VP.

Theorem 2.1. Assume that (2.11)-(2.17) hold. Then there exists a unique solution to Problem VP with the following regularity

$$
\boldsymbol{u} \in C^{1}([0, T] ; V), \quad \varphi \in C\left([0, T] ; W_{A}\right) .
$$

The proof of Theorem 2.1 is based on classical results of parabolic nonlinear variational equations and partial differential equations (see [22]).

\section{Fully Discrete approximations: ERror estimates}

We now introduce a finite element algorithm to approximate solutions to Problem VP and we derive an error estimate on them. Moreover, in order to simplify the writing we assume, in this section, that $\varphi_{A}=0$ (and then $\left.W_{A}=W\right)$. It is straightforward to extend the results presented below to a more general case.

The discretization of (2.18)-(2.19) is done as follows. First, we consider two finite dimensional spaces $V^{h} \subset V$ and $W^{h} \subset W$ approximating the spaces $V$ and $W$, respectively. $h>0$ denotes the spatial discretization parameter.

Remark 3.1. In the numerical simulations presented in the next section, $V^{h}$ and $W^{h}$ consist of continuous and piecewise affine functions, that is,

$$
\begin{aligned}
& V^{h}=\left\{\boldsymbol{w}^{h} \in[C(\bar{\Omega})]^{d} ; \boldsymbol{w}_{\left.\right|_{T r}}^{h} \in\left[P_{1}(T r)\right]^{d} \operatorname{Tr} \in \mathcal{T}^{h}, \quad \boldsymbol{w}^{h}=\mathbf{0} \text { on } \Gamma_{D}\right\}, \\
& W^{h}=\left\{\psi^{h} \in C(\bar{\Omega}) ; \psi_{\left.\right|_{T r}}^{h} \in P_{1}(T r) \quad \operatorname{Tr} \in \mathcal{T}^{h}, \quad \psi^{h}=0 \quad \text { on } \Gamma_{A}\right\},
\end{aligned}
$$

where $\Omega$ is assumed to be a polygonal domain, $\mathcal{T}^{h}$ denotes a finite element triangulation of $\bar{\Omega}$, and $P_{1}(\operatorname{Tr})$ represents the space of polynomials of global degree less or equal to one in $\mathrm{Tr}$.

To discretize the time derivatives, we use a uniform partition of $[0, T]$, denoted by $0=t_{0}<t_{1}<\ldots<t_{N}=T$, and let $k$ be the time step size, $k=T / N$. For a continuous function $f(t)$ let $f_{n}=f\left(t_{n}\right)$, and for a sequence $\left\{w_{n}\right\}_{n=0}^{N}$ we let $\delta w_{n}=\left(w_{n}-w_{n-1}\right) / k$ denote the divided differences.

In this section, no summation is assumed over a repeated index, and $c$ denotes a positive constant which depends on the problem data and the continuous solution, but it is independent of the discretization parameters $h$ and $k$.

Thus, using the backward Euler scheme, the fully discrete approximation of Problem VP is the following.

Problem VP $\mathbf{V P}^{h k}$. Find a discrete displacement field $\boldsymbol{u}^{h k}=\left\{\boldsymbol{u}_{n}^{h k}\right\}_{n=0}^{N} \subset V^{h}$ and a discrete electric potential field $\varphi^{h k}=\left\{\varphi_{n}^{h k}\right\}_{n=0}^{N} \subset W^{h}$ such that $\boldsymbol{u}_{0}^{h k}=\boldsymbol{u}_{0}^{h}$ and for all $n=1, \ldots, N$,

$$
\begin{aligned}
& \left(\mathcal{A} \varepsilon\left(\delta \boldsymbol{u}_{n}^{h k}\right), \boldsymbol{\varepsilon}\left(\boldsymbol{w}^{h}\right)\right)_{Q}+\left(\mathcal{B} \varepsilon\left(\boldsymbol{u}_{n}^{h k}\right), \boldsymbol{\varepsilon}\left(\boldsymbol{w}^{h}\right)\right)_{Q}+\left(\mathcal{E}^{*} \nabla \varphi_{n}^{h k}, \boldsymbol{\varepsilon}\left(\boldsymbol{w}^{h}\right)\right)_{Q} \\
& \quad+j\left(\boldsymbol{u}_{n}^{h k}, \boldsymbol{w}^{h}\right)=\left(\boldsymbol{f}_{n}, \boldsymbol{w}^{h}\right)_{V}, \quad \forall \boldsymbol{w}^{h} \in V^{h}, \\
& \left(\beta \nabla \varphi_{n}^{h k}, \nabla \psi^{h}\right)_{H}-\left(\mathcal{E} \boldsymbol{\varepsilon}\left(\boldsymbol{u}_{n}^{h k}\right), \nabla \psi^{h}\right)_{H}=\left(q_{n}, \psi^{h}\right)_{W}, \quad \forall \psi^{h} \in W^{h},
\end{aligned}
$$

where $\boldsymbol{u}_{0}^{h}$ is an appropriate approximation of the initial condition $\boldsymbol{u}_{0}$. 
We notice that the fully discrete problem $\mathrm{VP}^{h k}$ can be seen as a coupled system of variational equations. Using classical results of nonlinear variational equations (see [9]) we obtain that Problem VP ${ }^{h k}$ admits a unique solution $\boldsymbol{u}^{h k} \subset V^{h}$ and $\varphi^{h k} \subset W^{h}$, which we summarize in the following.

Theorem 3.2. Assume that (2.11)-(2.17) hold. Then there exists a unique solution to Problem $\mathrm{VP}^{h k}$.

Our interest in this section lies in estimating the numerical errors $\left\|\boldsymbol{u}_{n}-\boldsymbol{u}_{n}^{h k}\right\|_{V}$ and $\left\|\varphi_{n}-\varphi_{n}^{h k}\right\|_{W}$. We have the following main error estimates result.

Theorem 3.3. Assume that (2.11)-(2.17) hold. Let $(\boldsymbol{u}, \varphi)$ and $\left(\boldsymbol{u}^{h k}, \varphi^{h k}\right)$ denote the solutions to Problems VP and $\mathrm{VP}^{h k}$, respectively. Then, the following error estimates hold for all $\boldsymbol{w}^{h}=\left\{\boldsymbol{w}_{j}^{h}\right\}_{j=1}^{N} \subset V^{h}$ and $\psi^{h}=$ $\left\{\psi_{j}^{h}\right\}_{j=1}^{N} \subset W^{h}$,

$$
\begin{aligned}
& \max _{1 \leq n \leq N}\left\{\left\|\boldsymbol{u}_{n}-\boldsymbol{u}_{n}^{h k}\right\|_{V}^{2}+\left\|\varphi_{n}-\varphi_{n}^{h k}\right\|_{W}^{2}\right\} \leq c\left(\max _{1 \leq n \leq N}\left\|\varphi_{n}-\psi_{n}^{h}\right\|_{W}^{2}\right. \\
& +\sum_{j=1}^{N} k\left[\left\|\dot{\boldsymbol{u}}_{j}-\delta \boldsymbol{u}_{j}\right\|_{V}^{2}+\left\|\boldsymbol{u}_{j}-\boldsymbol{w}_{j}^{h}\right\|_{V}^{2}\right]+\max _{1 \leq n \leq N}\left\|\boldsymbol{u}_{n}-\boldsymbol{w}_{n}^{h}\right\|_{V}^{2} \\
& \left.+\left\|\boldsymbol{u}_{0}-\boldsymbol{u}_{0}^{h}\right\|_{V}^{2}+\frac{1}{k} \sum_{j=1}^{N-1}\left\|\boldsymbol{u}_{j}-\boldsymbol{w}_{j}^{h}-\left(\boldsymbol{u}_{j+1}-\boldsymbol{w}_{j+1}^{h}\right)\right\|_{V}^{2}\right) .
\end{aligned}
$$

Proof. First, let us obtain an error estimation on the electric potential. Then, taking (2.19) at time $t=t_{n}$ for $\psi=\psi^{h} \in W^{h}$ and subtracting it to (3.4) we obtain that

$$
\left(\beta \nabla\left(\varphi_{n}-\varphi_{n}^{h k}\right), \nabla \psi^{h}\right)_{H}-\left(\mathcal{E} \varepsilon\left(\boldsymbol{u}_{n}-\boldsymbol{u}_{n}^{h k}\right), \nabla \psi^{h}\right)_{H}=0, \quad \forall \psi^{h} \in W^{h} .
$$

Thus, we have

$$
\begin{aligned}
& \left(\beta \nabla\left(\varphi_{n}-\varphi_{n}^{h k}\right), \nabla\left(\varphi_{n}-\varphi_{n}^{h k}\right)\right)_{H}-\left(\mathcal{E} \boldsymbol{\varepsilon}\left(\boldsymbol{u}_{n}-\boldsymbol{u}_{n}^{h k}\right), \nabla\left(\varphi_{n}-\varphi_{n}^{h k}\right)\right)_{H} \\
& \quad=\left(\beta \nabla\left(\varphi_{n}-\varphi_{n}^{h k}\right), \nabla\left(\varphi_{n}-\psi^{h}\right)\right)_{H}-\left(\mathcal{E} \varepsilon\left(\boldsymbol{u}_{n}-\boldsymbol{u}_{n}^{h k}\right), \nabla\left(\varphi_{n}-\psi^{h}\right)\right)_{H},
\end{aligned}
$$

for all $\psi^{h} \in W^{h}$.

Applying the Cauchy's inequality

$$
a b \leq \epsilon a^{2}+\frac{1}{4 \epsilon} b^{2}, \quad a, b, \epsilon \in \mathbb{R}, \epsilon>0,
$$

and using properties (2.13) and (2.14), after some algebra we find that

$$
\left\|\varphi_{n}-\varphi_{n}^{h k}\right\|_{V}^{2} \leq c\left(\left\|\boldsymbol{u}_{n}-\boldsymbol{u}_{n}^{h k}\right\|_{V}^{2}+\left\|\varphi_{n}-\psi^{h}\right\|_{W}^{2}\right), \quad \forall \psi^{h} \in W^{h} .
$$

Secondly, we proceed now to estimate the numerical errors on the displacement field. We rewrite variational equation (2.18) at time $t=t_{n}$ for $\boldsymbol{w}=\boldsymbol{w}^{h} \in V^{h}$ and we subtract it to variational equation (3.3) to obtain

$$
\begin{gathered}
\left(\mathcal{A} \varepsilon\left(\dot{\boldsymbol{u}}_{n}-\delta \boldsymbol{u}_{n}^{h k}\right), \boldsymbol{\varepsilon}\left(\boldsymbol{w}^{h}\right)\right)_{Q}+\left(\mathcal{B} \varepsilon\left(\boldsymbol{u}_{n}-\boldsymbol{u}_{n}^{h k}\right)+\mathcal{E}^{*} \nabla\left(\varphi_{n}-\varphi_{n}^{h k}\right), \boldsymbol{\varepsilon}\left(\boldsymbol{w}^{h}\right)\right)_{Q} \\
+j\left(\boldsymbol{u}_{n}, \boldsymbol{w}^{h}\right)-j\left(\boldsymbol{u}_{n}^{h k}, \boldsymbol{w}^{h}\right)=0, \quad \forall \boldsymbol{w}^{h} \in V^{h}
\end{gathered}
$$

Therefore,

$$
\begin{aligned}
&\left(\mathcal{A} \varepsilon\left(\dot{\boldsymbol{u}}_{n}-\delta \boldsymbol{u}_{n}^{h k}\right), \boldsymbol{\varepsilon}\left(\boldsymbol{u}_{n}-\boldsymbol{u}_{n}^{h k}\right)\right)_{Q}+j\left(\boldsymbol{u}_{n}, \boldsymbol{u}_{n}-\boldsymbol{u}_{n}^{h k}\right)-j\left(\boldsymbol{u}_{n}^{h k}, \boldsymbol{u}_{n}-\boldsymbol{u}_{n}^{h k}\right) \\
& \quad+\left(\mathcal{B} \varepsilon\left(\boldsymbol{u}_{n}-\boldsymbol{u}_{n}^{h k}\right)+\mathcal{E}^{*} \nabla\left(\varphi_{n}-\varphi_{n}^{h k}\right), \boldsymbol{\varepsilon}\left(\boldsymbol{u}_{n}-\boldsymbol{u}_{n}^{h k}\right)\right)_{Q} \\
&=\left(\mathcal{A} \varepsilon\left(\dot{\boldsymbol{u}}_{n}-\delta \boldsymbol{u}_{n}^{h k}\right), \boldsymbol{\varepsilon}\left(\boldsymbol{u}_{n}-\boldsymbol{w}^{h}\right)\right)_{Q}+j\left(\boldsymbol{u}_{n}, \boldsymbol{u}_{n}-\boldsymbol{w}^{h}\right)-j\left(\boldsymbol{u}_{n}^{h k}, \boldsymbol{u}_{n}-\boldsymbol{w}^{h}\right) \\
& \quad+\left(\mathcal{B} \varepsilon\left(\boldsymbol{u}_{n}-\boldsymbol{u}_{n}^{h k}\right)+\mathcal{E}^{*} \nabla\left(\varphi_{n}-\varphi_{n}^{h k}\right), \varepsilon\left(\boldsymbol{u}_{n}-\boldsymbol{w}^{h}\right)\right)_{Q}, \quad \forall \boldsymbol{w}^{h} \in V^{h} .
\end{aligned}
$$


From properties (2.15), we have (see [8] for details),

$$
\begin{aligned}
& j\left(\boldsymbol{u}_{n}, \boldsymbol{u}_{n}-\boldsymbol{u}_{n}^{h k}\right)-j\left(\boldsymbol{u}_{n}^{h k}, \boldsymbol{u}_{n}-\boldsymbol{u}_{n}^{h k}\right) \geq 0, \\
& j\left(\boldsymbol{u}_{n}, \boldsymbol{u}_{n}-\boldsymbol{w}^{h}\right)-j\left(\boldsymbol{u}_{n}^{h k}, \boldsymbol{u}_{n}-\boldsymbol{w}^{h}\right) \leq c\left\|\boldsymbol{u}_{n}-\boldsymbol{u}_{n}^{h k}\right\|_{V}\left\|\boldsymbol{u}_{n}-\boldsymbol{w}^{h}\right\|_{V} .
\end{aligned}
$$

Using repeatedly inequality (3.6) and properties (2.11), (2.12), (2.13) and (2.15), after easy calculations it follows that

$$
\begin{aligned}
\left(\mathcal { A } \varepsilon \left(\delta \boldsymbol{u}_{n}-\right.\right. & \left.\left.\delta \boldsymbol{u}_{n}^{h k}\right), \boldsymbol{\varepsilon}\left(\boldsymbol{u}_{n}-\boldsymbol{u}_{n}^{h k}\right)\right)_{Q} \leq c\left(\left\|\dot{\boldsymbol{u}}_{n}-\delta \boldsymbol{u}_{n}\right\|_{V}^{2}+\epsilon\left\|\boldsymbol{u}_{n}-\boldsymbol{u}_{n}^{h k}\right\|_{V}^{2}\right. \\
& +\left\|\varphi_{n}-\varphi_{n}^{h k}\right\|_{W}^{2}+\left\|\boldsymbol{u}_{n}-\boldsymbol{w}^{h}\right\|_{V}^{2}+\left\|\boldsymbol{u}_{n}-\boldsymbol{u}_{n}^{h k}\right\|_{V}^{2} \\
& \left.+\left(\mathcal{A} \varepsilon\left(\delta \boldsymbol{u}_{n}-\delta \boldsymbol{u}_{n}^{h k}\right), \boldsymbol{\varepsilon}\left(\boldsymbol{u}_{n}-\boldsymbol{w}^{h}\right)\right)_{Q}\right), \quad \forall \boldsymbol{w}^{h} \in V^{h}
\end{aligned}
$$

where $\epsilon>0$, here and below, is assumed small enough and we denote $\delta \boldsymbol{u}_{n}=\left(\boldsymbol{u}_{n}-\boldsymbol{u}_{n-1}\right) / k$.

Keeping in mind that

$$
\left(\mathcal{A} \varepsilon\left(\delta \boldsymbol{u}_{n}-\delta \boldsymbol{u}_{n}^{h k}\right), \varepsilon\left(\boldsymbol{u}_{n}-\boldsymbol{u}_{n}^{h k}\right)\right)_{Q} \geq \frac{c}{2 k}\left(\left\|\boldsymbol{u}_{n}-\boldsymbol{u}_{n}^{h k}\right\|_{V}^{2}-\left\|\boldsymbol{u}_{n-1}-\boldsymbol{u}_{n-1}^{h k}\right\|_{V}^{2}\right),
$$

proceeding by induction it leads to the following inequality,

$$
\begin{aligned}
\| \boldsymbol{u}_{n}- & \boldsymbol{u}_{n}^{h k} \|_{V}^{2} \leq c \sum_{j=1}^{n} k\left(\left\|\dot{\boldsymbol{u}}_{j}-\delta \boldsymbol{u}_{j}\right\|_{V}^{2}+\left\|\varphi_{j}-\varphi_{j}^{h k}\right\|_{W}^{2}+\left\|\boldsymbol{u}_{j}-\boldsymbol{w}_{j}^{h}\right\|_{V}^{2}\right. \\
& \left.+\left\|\boldsymbol{u}_{j}-\boldsymbol{u}_{j}^{h k}\right\|_{V}^{2}+\left(\mathcal{A} \varepsilon\left(\delta \boldsymbol{u}_{j}-\delta \boldsymbol{u}_{j}^{h k}\right), \varepsilon\left(\boldsymbol{u}_{j}-\boldsymbol{w}_{j}^{h}\right)\right)_{Q}\right)+\left\|\boldsymbol{u}_{0}-\boldsymbol{u}_{0}^{h}\right\|_{V}^{2},
\end{aligned}
$$

for all $\boldsymbol{w}^{h}=\left\{\boldsymbol{w}_{j}^{h}\right\}_{j=0}^{n} \subset V^{h}$.

Since (see [11])

$$
\begin{aligned}
\sum_{j=1}^{n} k\left(\mathcal{A} \varepsilon\left(\delta \boldsymbol{u}_{j}-\delta \boldsymbol{u}_{j}^{h k}\right), \boldsymbol{\varepsilon}\left(\boldsymbol{u}_{j}-\boldsymbol{w}_{j}^{h}\right)\right)_{Q} \\
=\sum_{j=1}^{n}\left(\mathcal{A} \varepsilon\left(\boldsymbol{u}_{j}-\boldsymbol{u}_{j}^{h k}-\left(\boldsymbol{u}_{j-1}-\boldsymbol{u}_{j-1}^{h k}\right)\right), \boldsymbol{\varepsilon}\left(\boldsymbol{u}_{j}-\boldsymbol{w}_{j}^{h}\right)\right)_{Q} \\
\leq \epsilon\left\|\boldsymbol{u}_{n}-\boldsymbol{u}_{n}^{h k}\right\|_{V}^{2}+c\left\|\boldsymbol{u}_{n}-\boldsymbol{w}_{n}^{h}\right\|_{V}^{2}+c\left\|\boldsymbol{u}_{0}-\boldsymbol{u}_{0}^{h}\right\|_{V}^{2}+c\left\|\boldsymbol{u}_{1}-\boldsymbol{w}_{1}^{h}\right\|_{V}^{2} \\
\quad+c \sum_{j=1}^{n-1}\left\|\boldsymbol{u}_{j}-\boldsymbol{u}_{j}^{h k}\right\|_{V}\left\|\boldsymbol{u}_{j}-\boldsymbol{w}_{j}^{h}-\left(\boldsymbol{u}_{j+1}-\boldsymbol{w}_{j+1}^{h}\right)\right\|_{V},
\end{aligned}
$$

combining (3.7) and (3.8) and applying a discrete version of Gronwall's inequality (see [10] for details), we obtain (3.5).

We notice that the above error estimates are the basis for the analysis of the convergence rate of the algorithm. Thus, let $\Omega$ be a polyhedral domain and denote by $\mathcal{T}^{h}$ a triangulation of $\bar{\Omega}$ compatible with the partition of the boundary $\Gamma=\partial \Omega$ into $\Gamma_{D}, \Gamma_{F}, \Gamma_{C}$ on one hand, and on $\Gamma_{A}$ and $\Gamma_{B}$, on the other hand. Let $V^{h}$ and $W^{h}$ be defined by (3.1) and (3.2), respectively, and assume that the discrete initial condition $\boldsymbol{u}_{0}^{h}$ is obtained by

$$
\boldsymbol{u}_{0}^{h}=\Pi^{h} \boldsymbol{u}_{0}
$$

where $\Pi^{h}=\left(\pi^{h}\right)_{i=1}^{d}:[C(\bar{\Omega})]^{d} \rightarrow V^{h}$, and $\pi^{h}: C(\bar{\Omega}) \rightarrow W^{h}$ is the standard finite element interpolation operator (see, e.g., [6]).

Then, we have the following corollary which states the linear convergence of the algorithm under suitable regularity conditions. 
Corollary 3.4. Assume that (2.11)-(2.17) hold. Let $(\boldsymbol{u}, \varphi)$ and $\left(\boldsymbol{u}^{h k}, \varphi^{h k}\right)$ denote the solutions to Problems VP and $\mathrm{VP}^{\text {hk }}$, respectively, and let the discrete initial condition be given by (3.9). Under the following regularity conditions

$$
\boldsymbol{u} \in H^{2}(0, T ; V) \cap H^{1}\left([0, T] ;\left[H^{2}(\Omega)\right]^{d}\right), \quad \varphi \in C\left([0, T] ; H^{2}(\Omega)\right),
$$

the linear convergence of the algorithm is achieved, that is, there exists a positive constant $c>0$, independent of the discretization parameters $h$ and $k$, such that

$$
\max _{1 \leq n \leq N}\left\{\left\|\boldsymbol{u}_{n}-\boldsymbol{u}_{n}^{h k}\right\|_{V}+\left\|\varphi_{n}-\varphi_{n}^{h k}\right\|_{W}\right\} \leq c(h+k)
$$

Proof. We have the following approximation properties of the finite element spaces $V^{h}$ and $W^{h}$ (see [6]),

$$
\begin{aligned}
& \max _{1 \leq n \leq N} \inf _{\psi_{n}^{h} \in W^{h}}\left\|\varphi_{n}-\psi_{n}^{h}\right\|_{W} \leq \operatorname{ch}\|\varphi\|_{C\left([0, T] ; H^{2}(\Omega)\right)}, \\
& \max _{1 \leq n \leq N} \inf _{w_{n}^{h} \in V^{h}}\left\|\boldsymbol{u}_{n}-\boldsymbol{w}_{n}^{h}\right\|_{V} \leq \operatorname{ch}\|\boldsymbol{u}\|_{C\left([0, T] ;\left[H^{2}(\Omega)\right]^{d}\right)} .
\end{aligned}
$$

Moreover, from the definition of the finite element interpolation operator $\Pi^{h}$ it follows that

$$
\left\|\boldsymbol{u}_{0}-\boldsymbol{u}_{0}^{h}\right\|_{V} \leq \operatorname{ch}\|\boldsymbol{u}\|_{C\left([0, T] ;\left[H^{2}(\Omega)\right]^{d}\right)} .
$$

It is easy to check that

$$
\sum_{j=1}^{N} k\left\|\dot{\boldsymbol{u}}_{j}-\delta \boldsymbol{u}_{j}\right\|_{V}^{2} \leq c k^{2}\|\boldsymbol{u}\|_{H^{2}(0, T ; V)}^{2}
$$

Finally, we find that (see [11]),

$$
\frac{1}{k} \sum_{j=1}^{N-1}\left\|\boldsymbol{u}_{j}-\boldsymbol{w}_{j}^{h}-\left(\boldsymbol{u}_{j+1}-\boldsymbol{w}_{j+1}^{h}\right)\right\|_{V}^{2} \leq c h^{2}\|\boldsymbol{u}\|_{H^{1}\left(0, T ;\left[H^{2}(\Omega)\right]^{d}\right)}^{2} .
$$

Combining the previous estimates and (3.5) it leads to (3.11).

We notice that the regularity conditions (3.10) have not been proved yet. However, since the problem is quasistatic and the contact is produced with a deformable obstacle, it seems reasonable to assume them. Anyway, this is an open and interesting problem that we hope to address in the near future.

\section{NumERICAL RESUlts}

In order to recover the convergence results of the fully discrete method discussed in the previous section, some experiments have been done in the study of two two-dimensional test problems. First, in Section 4.1 we describe the algorithm used to solve Problem $\mathrm{VP}^{h k}$ and, secondly, in Sections 4.2 and 4.3, we consider two two-dimensional test problems in order to highlight the linear convergence obtained in Corollary 3.4, but also to describe some mechanical aspects of the frictionless viscoelastic piezoelectric contact behaviour.

\subsection{Numerical algorithm}

The algorithm, used in solving the fully discrete frictionless contact problem $\mathrm{VP}^{h k}$, is based on a backward Euler difference for the time derivatives and on a penalty approach (see [29] for more details) to simulate the normal compliance law. In order to give the solution algorithm, we have to introduce the expressions of 
the functions $\boldsymbol{w}^{h}, \boldsymbol{u}^{h}$ and $\boldsymbol{\delta} \boldsymbol{u}^{h}$ (resp. $\varphi^{h}$ and $\psi^{h}$ ) by considering theirs values at the $i^{\text {th }}$ nodes of $\mathcal{T}^{h}$ and the basis functions $\alpha^{i}$ (resp. $\gamma^{i}$ ) of the space $V^{h}$ (resp. $W^{h}$ ) for $i=1, \ldots, N_{t o t}$ ( $N_{\text {tot }}$ is the total number of nodes),

$$
\begin{aligned}
& \boldsymbol{w}^{h}=\sum_{i=1}^{N_{\text {tot }}} \boldsymbol{w}^{i} \alpha^{i}, \quad \boldsymbol{u}^{h}=\sum_{i=1}^{N_{\text {tot }}} \boldsymbol{u}^{i} \alpha^{i}, \quad \boldsymbol{\delta} \boldsymbol{u}^{h}=\sum_{i=1}^{N_{\text {tot }}} \boldsymbol{\delta} \boldsymbol{u}^{i} \alpha^{i}, \\
& \text { and } \quad \psi^{h}=\sum_{i=1}^{N_{\text {tot }}} \psi^{i} \gamma^{i}, \quad \varphi^{h}=\sum_{i=1}^{N_{\text {tot }}} \varphi^{i} \gamma^{i} .
\end{aligned}
$$

The penalty approach shows us that Problem $\mathrm{VP}^{h k}$ can be governed by the following system of nonlinear equations

$$
\tilde{\mathbf{A}}\left(\boldsymbol{\delta} \boldsymbol{u}_{n}\right)+\mathbf{G}\left(\boldsymbol{u}_{n}, \varphi_{n}\right)+\tilde{\mathcal{F}}\left(\boldsymbol{u}_{n}\right)=\mathbf{0} .
$$

The vectors $\boldsymbol{u}_{n}, \boldsymbol{\delta} \boldsymbol{u}_{n}$ and $\varphi_{n}$ represent respectively the generalized vectors defined as follows

$$
\boldsymbol{u}_{n}=\left\{\boldsymbol{u}_{n}^{i}\right\}_{i=1}^{N_{t o t}}, \boldsymbol{\delta} \boldsymbol{u}_{n}=\left\{\boldsymbol{\delta} \boldsymbol{u}_{n}^{i}\right\}_{i=1}^{N_{t o t}} \text { and } \varphi_{n}=\left\{\varphi_{n}^{i}\right\}_{i=1}^{N_{t o t}} .
$$

The generalized contact operator $\tilde{\mathcal{F}}\left(\boldsymbol{u}_{n}\right) \in \mathbb{R}^{d \times N_{\text {tot }}} \times \mathbb{R}^{N_{\text {tot }}}$ is defined by $\tilde{\mathcal{F}}\left(\boldsymbol{u}_{n}\right)=\left(\mathcal{F}\left(\boldsymbol{u}_{n}\right), \mathbf{0}_{\psi}\right)$, where the penalized contact operator $\mathcal{F}\left(\boldsymbol{u}_{n}\right)=c_{p} \operatorname{dist}\left(u_{\nu}\left(t_{n}\right)-g, \mathbb{R}^{+}\right) \boldsymbol{\nu}^{t} \in \mathbb{R}^{d \times N_{\text {tot }}}$ denotes the gradient of the penalized contact functional $\mathcal{L}^{h k}\left(\boldsymbol{u}_{n}\right)=\frac{c_{p}}{2} \int_{\Gamma_{C}} \operatorname{dist}^{2}\left(u_{\nu}\left(t_{n}\right)-g, \mathbb{R}^{+}\right) \mathrm{d} \Gamma$ in the direction $\boldsymbol{u}$,

$$
\left(\tilde{\mathcal{F}}\left(\boldsymbol{u}_{n}\right) \cdot(\boldsymbol{w}, \psi)\right)_{\mathbb{R}^{d \times N_{t o t} \times \mathbb{R}^{N} t o t}}=\left(\left(\mathcal{F}\left(\boldsymbol{u}_{n}\right), \mathbf{0}_{\psi}\right) \cdot(\boldsymbol{w}, \psi)\right)_{\mathbb{R}^{d \times N_{t o t}} \times \mathbb{R}^{N_{t o t}}}=\left(\nabla \boldsymbol{u}_{n} \mathcal{L}^{h k}\left(\boldsymbol{u}_{n}\right), \boldsymbol{w}\right)_{\mathbb{R}^{d \times N_{t o t}}}
$$

and $\mathbf{0}_{\psi}$ is the zero element of $\mathbb{R}^{N_{t o t}} . \quad c_{p}$ is the surface stiffness coefficient and so $1 / c_{p}$ is the deformability coefficient. In addition, the generalized viscous term $\tilde{\mathbf{A}}\left(\boldsymbol{\delta} \boldsymbol{u}_{n}\right) \in \mathbb{R}^{d \times N_{\text {tot }}} \times \mathbb{R}^{N_{t o t}}$ is defined by $\tilde{\mathbf{A}}\left(\boldsymbol{\delta} \boldsymbol{u}_{n}\right)=$ $\left(\mathbf{A}\left(\boldsymbol{\delta} \boldsymbol{u}_{n}\right), \mathbf{0}_{\psi}\right)$. Thus, the terms $\mathbf{A}\left(\boldsymbol{\delta} \boldsymbol{u}_{n}\right) \in \mathbb{R}^{d \times N_{\text {tot }}}$ and $\mathbf{G}\left(\boldsymbol{u}_{n}, \varphi_{n}\right)$ represent respectively the viscous term and the elastic-piezoelectric term given by

$$
\begin{aligned}
& \left(\left(\tilde{\mathbf{A}}\left(\boldsymbol{\delta} \boldsymbol{u}_{n}\right) \cdot(\boldsymbol{w}, \psi)\right)_{\mathbb{R}^{d \times N_{t o t} \times \mathbb{R}^{N_{t o t}}}}=\left(\left(\mathbf{A}\left(\boldsymbol{\delta} \boldsymbol{u}_{n}\right), \mathbf{0}_{\psi}\right) \cdot(\boldsymbol{w}, \psi)\right)_{\mathbb{R}^{d \times N_{t o t} \times \mathbb{R}^{N_{t o t}}}}\right. \\
& =\left(\mathbf{A}\left(\boldsymbol{\delta} \boldsymbol{u}_{n}\right) \cdot \boldsymbol{w}\right)_{\mathbb{R}^{d \times N_{t o t}}}=\left(\mathcal{A} \boldsymbol{\varepsilon}\left(\boldsymbol{\delta} \boldsymbol{u}_{n}\right), \boldsymbol{\varepsilon}\left(\boldsymbol{w}^{h}\right)\right)_{Q} \quad \forall \boldsymbol{w}^{h} \in V^{h}, \\
& \left(\mathbf{G}\left(\boldsymbol{u}_{n}, \varphi_{n}\right) \cdot(\boldsymbol{w}, \psi)\right)_{\mathbb{R}^{d \times N_{t o t} \times \mathbb{R}^{N_{t o t}}}}=\left(\mathcal{B} \varepsilon\left(\boldsymbol{u}_{n}\right), \boldsymbol{\varepsilon}\left(\boldsymbol{w}^{h}\right)\right)_{Q}+\left(\mathcal{E} \varepsilon\left(\boldsymbol{w}^{h}\right), \nabla \varphi_{n}\right)_{H}-\left(\boldsymbol{f}_{n}, \boldsymbol{w}^{h}\right)_{V} \\
& -\left(\mathcal{E} \varepsilon\left(\boldsymbol{u}_{n}\right), \nabla \psi^{h}\right)_{H}+\left(\beta \nabla \varphi_{n}, \nabla \psi^{h}\right)_{H}-\left(q_{n}, \psi^{h}\right)_{W} \forall \boldsymbol{w}^{h} \in V^{h}, \psi^{h} \in W^{h},
\end{aligned}
$$

where $\boldsymbol{w}\left(\right.$ resp. $\psi$ ) denotes the generalized vector constituted by the values $\boldsymbol{w}^{i}$ (resp. $\left.\psi^{i}\right)$ for $i=1, \ldots, N_{t o t}$. We remark that the volume and surface forces are contained in the term $\mathbf{G}\left(\boldsymbol{u}_{n}, \varphi_{n}\right)$.

The solution algorithm consists in a combination between the finite differences (backward Euler difference) and the linear iterations method (Newton method). To solve (4.3), at each time increment the variables $\left(\boldsymbol{u}_{n}, \varphi_{n}\right)$ are treated simultaneously through a Newton method and therefore in what follows we use $\mathbf{x}_{n}$ to denote the pair $\left(\boldsymbol{u}_{n}, \varphi_{n}\right)$. The algorithm that we used in the viscoelastic piezoelectric case can be developed in three steps which are the following:

\section{- A prediction step}

This step gives the initial displacement and the velocity by the following formula

$$
\varphi_{n+1}^{0}=\varphi_{n+1}, \quad \boldsymbol{u}_{n+1}^{0}=\boldsymbol{u}_{n+1} \quad \text { and } \quad \boldsymbol{\delta} \boldsymbol{u}_{n+1}^{0}=\boldsymbol{\delta} \boldsymbol{u}_{n}
$$




\section{- A Newton linearization step}

At an iteration $i$ of the Newton method, we have

$$
\begin{aligned}
\mathbf{x}_{n+1}^{i+1}=\mathbf{x}_{n+1}^{i}-\left(\frac{\boldsymbol{Q}_{n+1}^{i}}{k}+\right. & \left.\mathbf{K}_{n+1}^{i}+\boldsymbol{T}_{n+1}^{i}\right)^{-1} \\
& \times\left(\tilde{\mathbf{A}}\left(\boldsymbol{\delta} \boldsymbol{u}_{n+1}^{i}\right)+\mathbf{G}\left(\boldsymbol{u}_{n+1}^{i}, \varphi_{n+1}^{i}\right)+\tilde{\mathcal{F}}\left(\mathbf{u}_{n+1}^{i}\right)\right),
\end{aligned}
$$

with $\left.\boldsymbol{K}_{n+1}^{i}=D \boldsymbol{u}, \varphi \mathbf{G}\left(\boldsymbol{u}_{n+1}^{i}, \varphi_{n+1}^{i}\right), \boldsymbol{Q}_{n+1}^{i}=D \boldsymbol{u}, \varphi \tilde{\mathbf{A}}\left(\delta \boldsymbol{u}_{n+1}^{i}\right), \boldsymbol{T}_{n+1}^{i}=D \boldsymbol{u}, \varphi\right) \tilde{\mathcal{F}}\left(\boldsymbol{u}_{n+1}^{i}\right)$, and where $\mathbf{x}_{n+1}^{i+1}$ denotes the pair $\left(\boldsymbol{u}_{n+1}^{i+1}, \varphi_{n+1}^{i+1}\right) ; i$ and $n$ represent the Newton iteration index and the time index, respectively. Here, $D \boldsymbol{u}, \varphi \mathbf{G}, D \boldsymbol{u}, \varphi \tilde{\mathbf{A}}$ and $D \boldsymbol{u}, \varphi \tilde{\mathcal{F}}$ denote the differentials of the functions $\mathbf{G}, \tilde{\mathbf{A}}$ and $\tilde{\mathcal{F}}$ with respect to the variables $\boldsymbol{u}$ and $\varphi$. This leads us to solve the resulting linear system

$$
\begin{aligned}
\left(\frac{\boldsymbol{Q}_{n+1}^{i}}{k}+\right. & \left.\boldsymbol{K}_{n+1}^{i}+\boldsymbol{T}_{n+1}^{i}\right) \Delta \mathbf{x}^{i} \\
& =-\tilde{\mathbf{A}}\left(\boldsymbol{\delta} \boldsymbol{u}_{n+1}^{i}\right)-\mathbf{G}\left(\boldsymbol{u}_{n+1}^{i}, \varphi_{n+1}^{i}\right)-\tilde{\mathcal{F}}\left(\boldsymbol{\delta} \boldsymbol{u}_{n+1}^{i}\right),
\end{aligned}
$$

where $\Delta \mathbf{x}=\left(\Delta \boldsymbol{u}^{i}, \Delta \varphi^{i}\right)$ with $\Delta \boldsymbol{u}^{i}=\boldsymbol{u}_{n+1}^{i+1}-\boldsymbol{u}_{n+1}^{i}$ and $\Delta \varphi^{i}=\varphi_{n+1}^{i+1}-\varphi_{n+1}^{i}$. We solve the linear system of equations (4.6) by using a Conjugate Gradient Method with efficient preconditioners to overcome the poor conditioning of the matrix due to the penalized contact terms. In particular, we used specific incomplete LU factorization methods like the Element-By-Element preconditioner (see [1]). We notice that, in the case where the operators $\tilde{\mathbf{A}}$ and $\mathbf{G}$ are linear, the matrices $\boldsymbol{Q}_{n+1}^{i}$ and $\mathbf{K}_{n+1}^{i}$ do not change during the Newton iterations.

- A correction step

Once the system (4.6) is resolved, we update $\mathbf{x}_{n+1}^{i+1}$ and $\boldsymbol{\delta} \boldsymbol{u}_{n+1}^{i+1}$ by

$$
\mathbf{x}_{n+1}^{i+1}=\mathbf{x}_{n+1}^{i}+\Delta \mathbf{x}^{i} \quad \text { and } \quad \delta \boldsymbol{u}_{n+1}^{i+1}=\delta \boldsymbol{u}_{n+1}^{i}+\frac{\Delta \boldsymbol{u}^{i}}{k}
$$

For more considerations about Computational Contact Mechanics, see the recent monograph [29].

\subsection{First two-dimensional example}

In order to recover the theoretical numerical behaviour of the fully discrete scheme discussed in Section 3, we carry out some numerical simulations based on an academic viscoelastic piezoelectric contact problem with normal compliance law. To do that, we consider a piezoelectric body extending indefinitely in the first direction $X_{1}$ of a Cartesian coordinate frame $\left(O, X_{1}, X_{2}, X_{3}\right)$. The material used was assumed to be an academic isotropic piezoceramic with hexagonal symmetry like zinc oxide material (class $6 \mathrm{~mm}$ in the international classification [13]) but in which we introduce a viscous behaviour. In the crystallographic frame, the $X_{3}$-direction is a six-fold revolution symmetry axis and the $\left(X_{1} O X_{3}\right)$ and $\left(X_{2} O X_{3}\right)$ planes are mirrors. The electric and mechanical loads applied to the body are supposed to be constant along the $X_{1}$ direction. As a consequence, the fields $\mathbf{E}, \mathbf{D}, \varepsilon$ and $\boldsymbol{\sigma}$ turn out to be constant along $X_{1}$. In addition, we suppose that: $\varepsilon_{11}=0, \varepsilon_{12}=0$, $\varepsilon_{13}=0$ and $\mathbf{D}_{1}=0$; thus, we have to consider a plane problem. In the frame $\left(O, X_{2}, X_{3}\right)$, constitutive equations (2.1) and (2.2) can be written by using a compressed matrix notation instead of the tensor notation 
TABLE 1. Material viscoelastic constants of the considered piezoelectric body.

\begin{tabular}{|c|c|c|c|c|c|c|c|}
\hline \multicolumn{3}{|c|}{ Elastic (GPa) } & \multicolumn{4}{|c|}{ Viscoelastic (GPa $\cdot \mathrm{s})$} \\
\hline$b_{22}$ & $b_{23}$ & $b_{33}$ & $b_{44}$ & $a_{22}$ & $a_{23}$ & $a_{33}$ & $a_{44}$ \\
\hline 210 & 105 & 211 & 42.5 & 21 & 10.5 & 21.1 & 4.25 \\
\hline
\end{tabular}

TABLE 2. Material electric constants of the considered piezoelectric body.

\begin{tabular}{|c|c|c|c|c|}
\hline \multicolumn{2}{|c|}{ Piezoelectric $\left(\mathrm{C} \cdot \mathrm{m}^{-2}\right)$} & \multicolumn{2}{c|}{ Permittivity $\left(\mathrm{C}^{2} \cdot \mathrm{N}^{-1} \cdot \mathrm{m}^{-2}\right)$} \\
\hline$e_{32}$ & $e_{33}$ & $e_{24}$ & $\beta_{22} / \epsilon_{0}$ & $\beta_{33} / \epsilon_{0}$ \\
\hline-0.61 & 1.14 & -0.59 & -8.3 & -8.8 \\
\hline
\end{tabular}

as follows,

$$
\begin{aligned}
{\left[\begin{array}{c}
\sigma_{22} \\
\sigma_{33} \\
\sigma_{23} \\
D_{2} \\
D_{3}
\end{array}\right]=} & {\left[\begin{array}{ccccc}
b_{22} & b_{23} & 0 & 0 & e_{32} \\
b_{23} & b_{33} & 0 & 0 & e_{33} \\
0 & 0 & b_{44} & e_{24} & 0 \\
0 & 0 & e_{24} & -\beta_{22} & 0 \\
e_{32} & e_{33} & 0 & 0 & -\beta_{33}
\end{array}\right]\left[\begin{array}{c}
\varepsilon_{22} \\
\varepsilon_{33} \\
2 \varepsilon_{23} \\
-E_{2} \\
-E_{3}
\end{array}\right] } \\
& +\left[\begin{array}{ccccc}
a_{22} & a_{23} & 0 & 0 & 0 \\
a_{23} & a_{33} & 0 & 0 & 0 \\
0 & 0 & a_{44} & 0 & 0 \\
0 & 0 & 0 & 0 & 0 \\
0 & 0 & 0 & 0 & 0
\end{array}\right]\left[\begin{array}{c}
\dot{\varepsilon}_{22} \\
\dot{\varepsilon}_{33} \\
2 \dot{\varepsilon}_{23} \\
-E_{2} \\
-E_{3}
\end{array}\right]
\end{aligned}
$$

where $\dot{\varepsilon}_{i j}=\frac{1}{2}\left(\frac{\partial \dot{u}_{i}}{\partial x_{j}}+\frac{\partial \dot{u}_{j}}{\partial x_{i}}\right)$. In equation (4.7), taking advantage of the symmetries of the mechanical tensors, the passage of the fourth-order viscosity and elastic tensors $\left(b_{i j k l}\right.$ and $\left.a_{i j k l}\right)$ to the second-order tensors (with the matrix notation: $b_{p q}$ and $\left.a_{p q}\right)$ is done by using the following identifications:

$$
b_{i j k l} \equiv b_{p q}=\left(\begin{array}{ccc}
b_{22} & b_{23} & 0 \\
b_{23} & b_{33} & 0 \\
0 & 0 & b_{44}
\end{array}\right) \quad \text { and } \quad a_{i j k l} \equiv a_{p q}=\left(\begin{array}{ccc}
a_{22} & a_{23} & 0 \\
a_{23} & a_{33} & 0 \\
0 & 0 & a_{44}
\end{array}\right)
$$

with the following notation:

$$
\begin{aligned}
& i j \text { or } k l=22 \quad \longrightarrow \quad p \text { or } q=2, \\
& i j \text { or } k l=33 \longrightarrow p \text { or } q=3 \text {, } \\
& i j \text { or } k l=23 \text { or } 32 \quad \longrightarrow \quad p \text { or } q=4 \text {. }
\end{aligned}
$$

In the same way for the third order piezoelectric tensor, we have,

$$
e_{i j k} \equiv e_{i q}=\left(\begin{array}{ccc}
0 & 0 & e_{24} \\
e_{32} & e_{33} & 0
\end{array}\right) \quad \text { with } \begin{aligned}
j k=22 & \longrightarrow q=2, \\
j k=33 & \longrightarrow q=3, \\
j k=23 \text { or } 32 & \longrightarrow q=4 .
\end{aligned}
$$

The coefficient values are given in Tables 1 and 2. The permittivity constant of the vacuum $\epsilon_{0}=8.885 \times$ $10^{-12} \mathrm{C}^{2} \cdot \mathrm{N}^{-1} \cdot \mathrm{m}^{-2}$.

As a first two-dimensional example, we consider the problem depicted in Figure 2, where a square body is in contact with a foundation. The domain $\Omega=(0,1) \times(0,1)$ is a cross-section of a three-dimensional square 


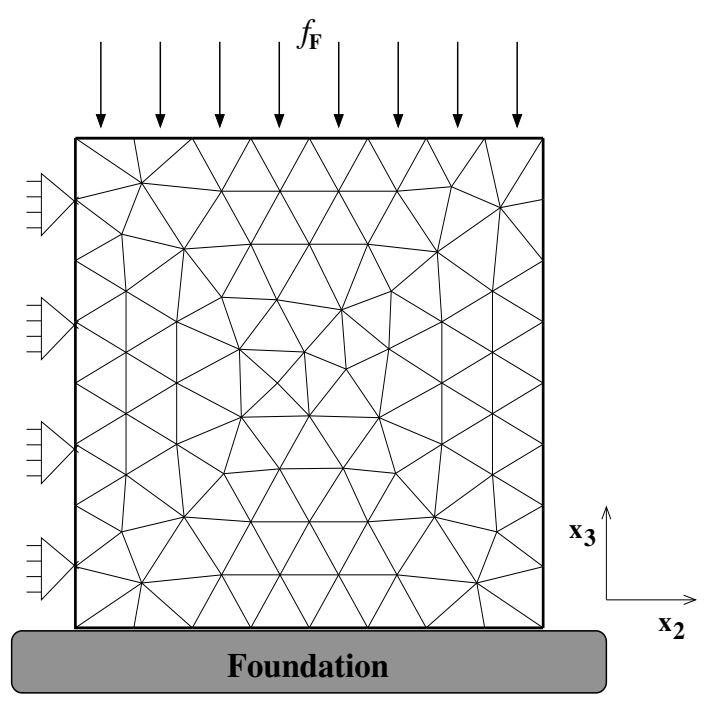

Figure 2. Discretization of the body in contact with a foundation.

body clamped on $\Gamma_{D}=\Gamma_{A}=\{0\} \times[0,1]$ and the electric potential is equal to zero there. On the remaining boundary $\Gamma_{B}$ of $\Omega$, we assume that $q_{F}=0 \mathrm{C} \cdot \mathrm{m}^{-2}$. Let $\Gamma_{F}=(\{1\} \times[0,1]) \cup([0,1] \times\{1\})$. The body is subjected to the action of surface tractions acting on $[0,1] \times\{1\}$, i.e., $\boldsymbol{f}_{F}=(0,-10) \mathrm{N} \cdot \mathrm{m}^{-2}$, while the part $\{1\} \times[0,1]$ is free. The body is in frictionless contact with an insulator foundation with normal compliance, in the form with a zero gap function, on $\Gamma_{C}=[0,1] \times\{0\}$. Finally, we assume that there are no body forces and no electric charges, i.e. $\boldsymbol{f}_{0}=\mathbf{0} \mathrm{N} \cdot \mathrm{m}^{3}, q_{0}=0 \mathrm{C} \cdot \mathrm{m}^{-3}$, and we use the following initial condition $\boldsymbol{u}_{0}=\mathbf{0} \mathrm{m}$.

To see the convergence behaviour of the fully discrete scheme, we compute a sequence of numerical solutions based on uniform partitions of the time interval $[0,1]$, and uniform triangulations of the domain $[0,1] \times[0,1]$ of the type shown in Figure 2 which represents a coarse discretization $(h=1 / 8)$. Then, we provide the estimated error values for several discretization parameters $h$ and $k$. Here, the sides of the square are divided into $1 / h$ equal parts. We start with $h=1 / 2$ and $k=1 / 2$ which are successively halved. The numerical solution corresponding to $h=1 / 256$ and $k=1 / 256$ is taken as the "exact" solution, which is used to compute the errors of the numerical solutions with larger values of $h$ and $k$; this finer discretization corresponds to a problem with around 222900 degrees of freedom. The linear asymptotic convergence behaviour obtained in Corollary 3.4 is almost observed (see Fig. 3).

\subsection{Second two-dimensional example}

As a second two-dimensional example of Problem $\mathrm{P}$, we consider the body $\Omega$ with the boundary $\Gamma$ which can come into contact with a deformable foundation (see the setting shown on the left-hand side of Fig. 4). To fix the geometry we set the points $P_{1}=(0,1), P_{2}=(1,0), P_{3}=(3,1), P_{4}=(1.5,1), P_{5}=(1,1.5)$ and $P_{6}=(1,4)$. We define $\Gamma_{B}=\Gamma_{C}=\left[P_{1}, P_{2}\right], \Gamma_{D}=\left[P_{4}, P_{5}\right]$ and $\Gamma_{F}=\Gamma \backslash\left(\Gamma_{C} \cup \Gamma_{D}\right) ;\left\{X_{2}, X_{3}\right\}$ denotes the canonical orthonormal basis. Here, we use as material the viscoelastic piezoelectric body whose constants are given in Tables 1 and 2 . We suppose that the body is clamped on $\Gamma_{D}$ and we employ the following data:

$$
\begin{aligned}
& \boldsymbol{f}_{F}=\mathbf{0 N} \cdot \mathrm{m}^{-2}, \quad \boldsymbol{f}_{0}=\mathbf{0 N} \cdot \mathrm{m}^{-3}, \quad q_{0}=0 \mathrm{C} \cdot \mathrm{m}^{-3}, \\
& \varphi_{A}=\left\{\begin{array}{cc}
20 \mathrm{~V} & \text { on }\left[P_{3}, P_{4}\right], \\
0 \mathrm{~V} & \text { on }\left[P_{5}, P_{6}\right],
\end{array}\right. \\
& g=10^{-4} \mathrm{~m}, \quad T=10 \mathrm{~s}, \quad \boldsymbol{u}_{0}=\mathbf{0} \mathrm{m} .
\end{aligned}
$$




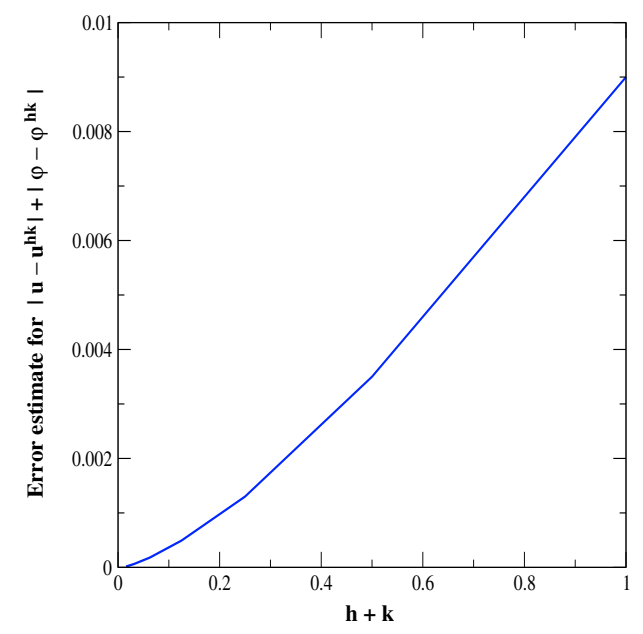

Figure 3. Estimated errors for the first two-dimensional example.
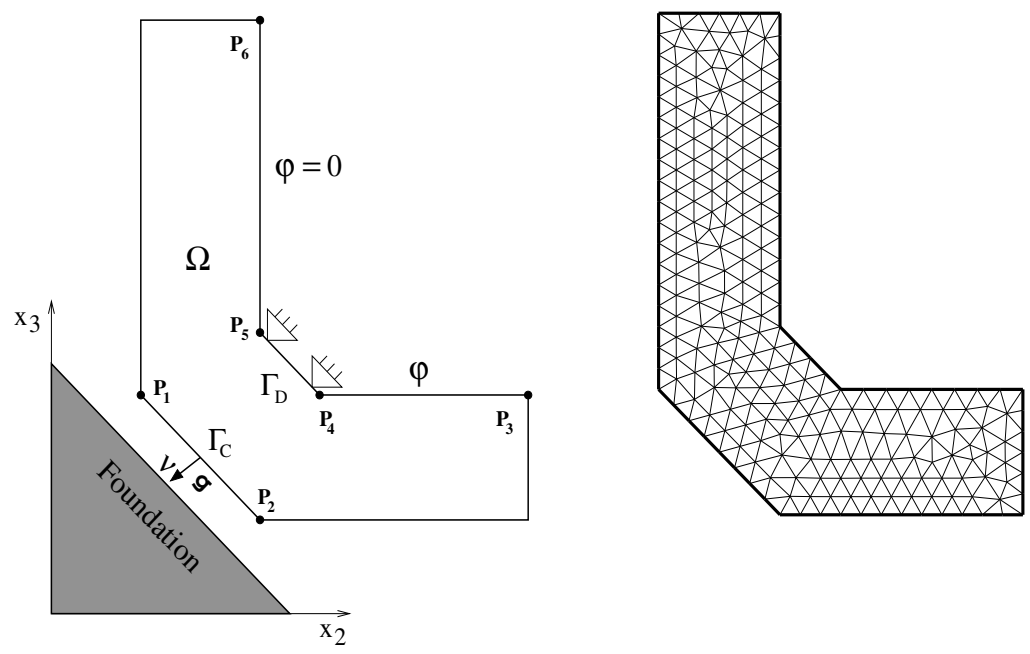

Figure 4. Problem setting (left) and the discretization of the body (right).

This physical setting permits to show the inverse piezoelectric effect that corresponds to the appearance of strain or stress in the body due to the action of the electric field. This example represents a contactor stimulated by an electric field.

We suppose that the time interval $[0,1]$ is discretized with a uniform partition. The picture on the right-hand side of Figure 4 shows a uniform triangulation of the domain $\Omega$. According to Figure 5 , it can be seen that the action of the difference of the electric field on $\left[P_{3}, P_{4}\right]$ and $\left[P_{5}, P_{6}\right]$ induces a deformation of the body. That results in to make come into contact the body with the foundation on $\Gamma_{C}$. Indeed, we remark that some contact nodes are in slip case and the contact forces are following the exterior normal on $\Gamma_{C}$. This is due to the fact that the problem is frictionless. Moreover, Figure 6 shows the distribution of the electric displacement field and the electric potential in the body. We can notice a certain correspondence between the distribution of the electric displacement field and the viscoelastic constraints presented in Figure 7. This happens since the higher values of the electric field are localised on the zones where the viscoelastic constraints are stronger. 


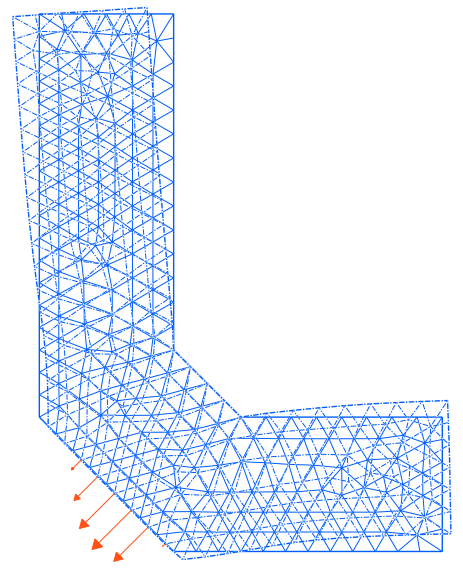

FiguRE 5. Initial and amplified deformed mesh with contact interface forces in the viscoelastic piezoelectric case.

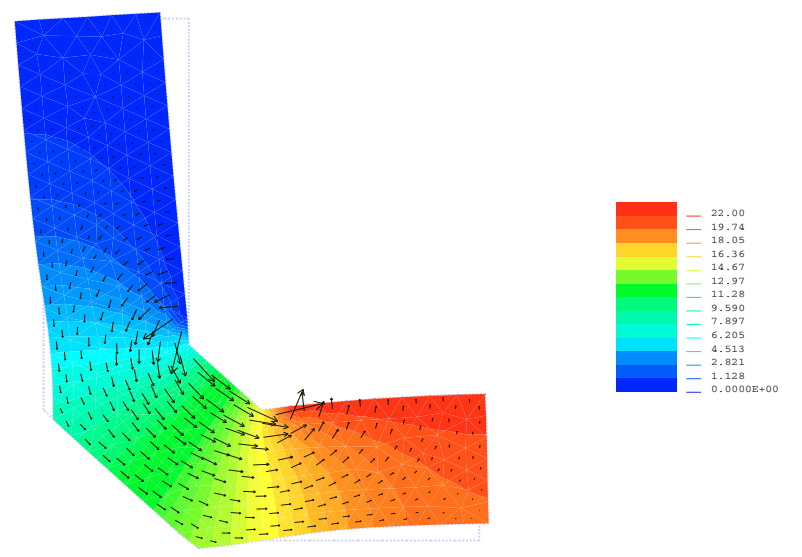

FiguRE 6. The electric displacement field (arrows) and the electric potential (colours) in the deformed configuration.
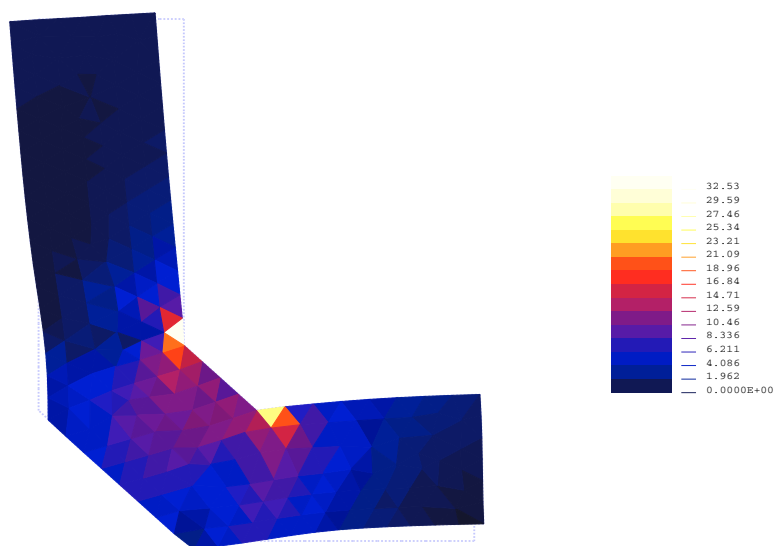

FIGURE 7. Initial boundary and the viscoelastic constraints in the deformed configuration. 


\section{REFERENCES}

[1] P. Alart, M. Barboteu and F. Lebon, Solution of frictional contact problems by an EBE preconditioner. Comput. Mech. 20 (1997) 370-378.

[2] F. Auricchio, P. Bisegna and C. Lovadina, Finite element approximation of piezoelectric plates. Internat. J. Numer. Methods Engrg. 50 (2001) 1469-1499.

[3] M. Barboteu, J.R. Fernández and Y. Ouafik, Numerical analysis of two frictionless elastic-piezoelectric contact problems. J. Math. Anal. Appl. 339 (2008) 905-917.

[4] R.C. Batra and J.S. Yang, Saint-Venant's principle in linear piezoelectricity. J. Elasticity 38 (1995) 209-218.

[5] P. Bisegna, F. Lebon and F. Maceri, The unilateral frictional contact of a piezoelectric body with a rigid support, in Contact mechanics (Praia da Consolação, 2001), Solid Mech. Appl. 103, Kluwer Acad. Publ., Dordrecht (2002) 347-354.

[6] P.G. Ciarlet, The finite element method for elliptic problems, in Handbook of Numerical Analysis, Vol. II, P.G. Ciarlet and J.L. Lions Eds., North Holland (1991) 17-352.

[7] G. Duvaut and J.L. Lions, Inequalities in Mechanics and Physics. Springer Verlag, Berlin (1976).

[8] J.R. Fernández, M. Sofonea and J.M. Viaño, A frictionless contact problem for elastic-viscoplastic materials with normal compliance: Numerical analysis and computational experiments. Numer. Math. 90 (2002) 689-719.

[9] R. Glowinski, Numerical Methods for Nonlinear Variational Problems. Springer, New York (1984).

[10] W. Han and M. Sofonea, Quasistatic Contact Problems in Viscoelasticity and Viscoplasticity. American Mathematical SocietyInternational Press (2002).

[11] W. Han, M. Shillor and M. Sofonea, Variational and numerical analysis of a quasistatic viscoelastic problem with normal compliance, friction and damage. J. Comput. Appl. Math. 137 (2001) 377-398.

[12] S. Hüeber, A. Matei and B.I. Wohlmuth, A mixed variational formulation and an optimal a priori error estimate for a frictional contact problem in elasto-piezoelectricity. Bull. Math. Soc. Sci. Math. Roumanie 48 (2005) 209-232.

[13] T. Ideka, Fundamentals of Piezoelectricity. Oxford University Press, Oxford (1990).

[14] A. Klarbring, A. Mikelić and M. Shillor, Frictional contact problems with normal compliance. Internat. J. Engrg. Sci. 26 (1988) 811-832.

[15] F. Maceri and B. Bisegna, The unilateral frictionless contact of a piezoelectric body with a rigid support. Math. Comput. Modelling 28 (1998) 19-28.

[16] J.A.C. Martins and J.T. Oden, Existence and uniqueness results for dynamic contact problems with nonlinear normal and friction interface laws. Nonlinear Anal. 11 (1987) 407-428.

[17] R.D. Mindlin, Polarisation gradient in elastic dielectrics. Internat. J. Solids Structures 4 (1968) 637-663.

[18] R.D. Mindlin, Continuum and lattice theories of influence of electromechanical coupling on capacitance of thin dielectric films. Internat. J. Solids Structures 5 (1969) 1197-1213.

[19] R.D. Mindlin, Elasticity, piezoelasticity and crystal lattice dynamics. J. Elasticity 4 (1972) 217-280.

[20] A. Morro and B. Straughan, A uniqueness theorem in the dynamical theory of piezoelectricity. Math. Methods Appl. Sci. 14 (1991) 295-299.

[21] Y. Ouafik, A piezoelectric body in frictional contact. Bull. Math. Soc. Sci. Math. Roumanie 48 (2005) 233-242.

[22] M. Sofonea and E.-H. Essoufi, Quasistatic frictional contact of a viscoelastic piezoelectric body. Adv. Math. Sci. Appl. 14 (2004) $25-40$.

[23] M. Sofonea and E.-H. Essoufi, A piezoelectric contact problem with slip dependent coefficient of friction. Math. Model. Anal. 9 (2004) 229-242.

[24] M. Sofonea and Y. Ouafik, A piezoelectric contact problem with normal compliance. Appl. Math. 32 (2005) 425-442.

[25] R.A. Toupin, The elastic dielectrics. J. Rational Mech. Anal. 5 (1956) 849-915.

[26] R.A. Toupin, Stress tensors in elastic dielectrics. Arch. Rational Mech. Anal. 5 (1960) 440-452.

[27] R.A. Toupin, A dynamical theory of elastic dielectrics. Internat. J. Engrg. Sci. 1 (1963) 101-126.

[28] N. Turbé and G.A. Maugin, On the linear piezoelectricity of composite materials. Math. Methods Appl. Sci. 14 (1991) $403-412$.

[29] P. Wriggers, Computational Contact Mechanics. Wiley-Verlag (2002). 